\title{
Dieldrin resistance in Lucilia cuprina (the Australian sheep blowfly): chance, selection and response
}

\author{
MEGAN SCOTT, KYLIE DIWELL \& JOHN A. McKENZIE* \\ Department of Genetics, The University of Melbourne, Victoria 3010, Australia
}

\begin{abstract}
Discrete-generation population cages of Lucilia cuprina were initiated with dieldrin-resistant allele $(R d l)$ frequencies of 1 or $5 \%$ and maintained for 17 generations on media with concentrations of dieldrin in the range $0-0.006 \%(\mathrm{w} / \mathrm{v})$. The probability of the initial establishment of the $R d l$ allele in a population was consistently greater at the $5 \%$ frequency and dependent on the concentration of dieldrin in the medium for both starting frequencies. Once the resistant allele was established responses to selection were concentration-dependent. It was concluded that in the absence of dieldrin the susceptible allele was selectively favoured, at $0.00005 \%(\mathrm{w} / \mathrm{v})$ concentration selection and random genetic drift influenced changes in allele frequency and at concentrations above this the $R d l$ allele was at a selective advantage. Fixation of $R d l$ occurred at the higher concentrations. The influence of random genetic drift and selection on the genetic response during the evolution of insecticide resistance is discussed.
\end{abstract}

Keywords: dieldrin resistance, founding events, Lucilia cuprina, random genetic drift, selection.

\section{Introduction}

As a general thesis for the evolution of resistance to pesticides it has been proposed that monogenic responses for rare mutations will be favoured preferentially if selection acts outside the initial phenotypic distribution of susceptible individuals (that is, above the lethal concentration for $100 \%$ of all individuals in the distribution, the $\left.\mathrm{LC}_{100}\right)$. Conversely, a polygenic response is more likely when selection acts within the initial distribution (at concentrations less than the $\mathrm{LC}_{100}$ ) as any genetic differences between individuals of a continuous distribution are assumed to be polygenically based (Whitten \& McKenzie, 1982; Macnair, 1991; McKenzie \& Batterham, 1994, 1998; Gressel, 1995; McKenzie, 1996). However, in the field such clear distinctions may not occur as the fitness differentials between genotypes depend on decay rates and the exposure characteristics of pesticides to life-history stages (McKenzie \& Whitten, 1982; Daly et al., 1988; Follett et al., 1993; Carrière et al., 1994).

The key question to resolve for the basis of monogenic/polygenic response is the conditions under which selection for rare resistant variants can occur. There is

*Correspondence. E-mail: j.mc_kenzie@genetics.unimelb.edu.au evidence that in some circumstances selection within the distribution of susceptibles, at concentrations less than the $\mathrm{LC}_{100}$, may screen for monogenic variants if these variants are present within the initial population (Roush \& Hoy, 1981; Heather, 1986; Martin \& McKenzie, 1990). However, if there is a fitness 'cost' associated with the variant and if the range of pesticide concentrations to which the population is exposed does not specifically select for the variant, it may be lost from the population (Roush \& McKenzie, 1987).

It is typically assumed that in the absence of the pesticide resistant individuals are selected against because of pleiotropic effects of resistant genotypes. It should be noted, however, that differences in relative fitness may be variable and not necessarily large (Roush \& McKenzie, 1987; Bergelson \& Purrington, 1996; McKenzie, 1996). Identifying the conditions that determine the balance between selection for polygenic and monogenic responses is potentially of critical importance to the determination of resistance management strategies (Gressel, 1995; McKenzie, 1996; Denholm et al., 1999) and, more generally, to the genetic structure of populations (Macnair, 1991; Orr \& Coyne, 1992).

The relative influence of random genetic drift, particularly during founding events, and selection on that genetic structure is also of considerable importance 
(Kimura, 1983; Endler, 1986). The initial frequency of a resistant allele in a population should be considered in this context. With respect to the evolution of pesticide resistance the selection coefficients of resistance genotypes are typically regarded as being sufficiently large to preclude the relevance of random genetic drift. For this reason, the impact of chance on the initial establishment of resistant alleles remains a neglected area of research (Tabashnik, 1990). However, when the frequency of resistant alleles is low in a population, stochastic events may potentially influence evolutionary outcomes (Whitten \& McKenzie, 1982). It should be noted, however, that the relative importance of selection and random genetic drift to the genetic response of populations exposed to different concentrations of insecticides has not been investigated systematically.

Empirical consideration of the problem requires a model system in which: the organism is amenable to laboratory analysis; there is a strong foundation of toxicological, genetic and relative fitness information relating to the evolution of resistance; and in which polygenic and monogenic responses can be distinguished. A number of systems meet these requirements (McKenzie, 1996). The dieldrin resistance system of the Australian sheep blowfly, Lucilia cuprina, is one.

Resistance to dieldrin developed within two years of the introduction of the insecticide for blowfly control. Resistance in natural populations is controlled by allelic substitution at a single genetic locus $(R d l)$ with selection for resistance occurring over a range of concentrations of dieldrin (McKenzie \& Whitten, 1982). Selection for susceptibility is observed in the absence of the insecticide (Whitten et al., 1980; McKenzie, 1990, 1996). Genotypes can be unambiguously identified on the basis of discriminating concentrations of the chemical (Whitten et al., 1980; Smyth et al., 1992). Population cage analyses have proved effective in demonstrating fitness differences and changes in allele frequency in L. cuprina (McKenzie, 1996).

This system is therefore ideally placed to allow the analysis of the effect of initial allele frequencies and the selective regime on the establishment of resistance in a population and subsequent allelic frequency changes. It therefore provides the opportunity to understand the nature of the genetic response for insecticide resistance and, by analogy, for more general evolutionary systems.

\section{Materials and methods}

\section{Strains}

The strains of L. cuprina used in this study were a standard laboratory susceptible strain (SWT, $+/+$ at the $R d l$ locus) and a dieldrin-resistant strain $(R d l / R d l)$ where the $R d l$ allele was placed in the genetic background of the susceptible strain (SWT). The $R d l / R d l$ strain used in these experiments was constructed from a dieldrinresistant strain that was isolated initially following EMS mutagenesis and selection for dieldrin resistance in SWT (Smyth et al., 1992). The resistant strain thus isolated, which carried an identical $R d l$ allele to that found in natural populations (McKenzie \& Batterham, 1998), was backcrossed to SWT for 10 generations to further ensure that the resistant $(R d l / R d l)$ and susceptible $(+/+)$ strains used in this study shared a common genetic background. This was achieved by selecting heterozygous females with a discriminating concentration of dieldrin $[0.01 \%(\mathrm{w} / \mathrm{v})]$ at each generation of backcrossing. At the conclusion of the backcrossing regime heterozygotes were intercrossed and $R d l / R d l$ homozygotes selected by a second appropriate discriminating concentration $[0.1 \%(\mathrm{w} / \mathrm{v})]$ (Smyth et al., 1992).

\section{Establishment of population cages}

At generation 0 , population cages were initiated with 50 males and 50 virgin females. Flies were of genotype $R d l /+$ or $+/+$ with the number of heterozygotes (one or five of each sex) determining the starting allele frequency ( $1 \%$ or $5 \%$ ) of the $R d l$ allele in each founding population.

Discrete-generation population cages were maintained under standard laboratory conditions with larvae of a particular cage being raised on meat meal medium with dieldrin concentrations of $0,0.00005,0.0001$, $0.0002,0.0004,0.0006,0.004$ or $0.006 \%$ (w/v) dieldrin. This range of concentrations is lethal to between 0 and $100 \%$ of susceptibles (Smyth et al., 1992). Cages were initiated at each starting frequency on each concentration until the $R d l$ allele was established in generation one in three trials. The $R d l$ allele was deemed established in a population if at least one resistant phenotype was recorded in the first generation. To ascertain the presence of the $R d l$ allele, all flies of that generation were tested after generation two was established.

The number of attempts necessary to establish the three successful trials was recorded and the percentage establishment success calculated. Thus, if six attempts were necessary to establish the $R d l$ allele in three trials of a certain experimental condition the percentage establishment success was 50\%. Approximately 100 larvae were used to establish each generation of each cage throughout the experiment.

\section{Estimation of frequency of resistant allele}

Twenty males from each cage were tested in each generation using standard toxicological procedures 
(Smyth et al., 1992). Males were tested with $0.5 \mu \mathrm{L}$ of $0.01 \%(\mathrm{w} / \mathrm{v})$ dieldrin, which is lethal to $+/+$ flies. Mortality was recorded after $24 \mathrm{~h}$ to identify $+/+$ flies and survivors treated with $0.1 \%(\mathrm{w} / \mathrm{v})$ dieldrin. Survivors after $24 \mathrm{~h}$ were of genotype $R d l / R d l$, dead flies, $R d l /+$ (Smyth et al., 1992). At each generation, control flies of known genotype were tested to ensure confidence in the discriminating concentrations and the estimates of the allelic frequencies in the population cages. Estimates of allelic frequency were only made when sufficient flies were available for testing.

\section{Results}

\section{Population cages}

The probability of the $R d l$ allele being initially established in the population is a function of both allelic frequency and the concentration of dieldrin on which the population is maintained (Fig. 1). There is a significantly $\left(F_{1,7}=47.8, P<0.001\right)$ higher probability of establishment in populations when the $R d l$ allele is at an initial frequency of $5 \%$ in founders than when the frequency is $1 \%$. The concentration of dieldrin in the medium also influences the likelihood of the $R d l$ allele being established in the population $\left(F_{7,7}=10.6\right.$, $P<0.01)$. Least-significant difference analysis shows that for both initial $R d l$ frequencies, populations maintained on 0.004 and $0.006 \%(\mathrm{w} / \mathrm{v})$ dieldrin have a lower chance of establishment than populations at other concentrations (Fig. 1).

After the initial establishment of the $R d l$ allele in a population, generally similar average trends in allelic frequency were observed for populations maintained at the same concentration, irrespective of the frequency of the $R d l$ allele in the founders (Fig. 2). Thus, in the absence of dieldrin, all populations eventually fix for the susceptible allele, although this occurs more quickly in populations in which the frequency of $R d l$ in the founders was lower.

There is evidence of selection of the $R d l$ allele at concentrations above $0.00005 \%(\mathrm{w} / \mathrm{v})$ dieldrin. The intensity of selection is a function of the concentration of dieldrin in the media on which populations are raised. Thus, by generation 17, populations are at, or near, fixation of the $R d l$ allele for concentrations of $0.0004 \%$ $(\mathrm{w} / \mathrm{v})$ and above, at an average frequency of approximately 0.85 at $0.0002 \%(\mathrm{w} / \mathrm{v})$ and in the range $0.50-0.80$ for populations raised at $0.0001 \% \quad(\mathrm{w} / \mathrm{v})$ dieldrin (Fig. 2). In all cases the gradient of the regression line of $R d l$ allele frequency on generation number is significantly $\left(t_{15}>4.07, P<0.001\right)$ different from zero.

At concentration $0.00005 \%(\mathrm{w} / \mathrm{v})$ dieldrin differences are observed between population cages initiated at $1 \%$ and $5 \% R d l$ allele frequencies. At the $1 \%$ frequency there is an increase in $R d l$ frequency with generation, the gradient of the regression line being significantly $\left(t_{15}=5.63, P<0.001\right)$ different from zero. In the population cages initiated at the $5 \% R d l$ allele frequency there was no association between allele frequency and generation number $\left(t_{15}=0.66,0.5>P>0.4\right)$. The susceptible allele fixed in one trial of these cages.

In spite of the differences observed in cages maintained at concentration $0.00005 \%(\mathrm{w} / \mathrm{v})$ dieldrin, the general similarity of outcome 17 generations after establishment at the two initial frequencies is emphasized by
Fig. 1 The percentage establishment success of the $R d l$ allele in population cages at each concentration $[\%(w / v)]$ of dieldrin for populations with the $R d l$ allele frequencies of 1 or $5 \%$ in generation 0 . The percentage is based on the number of attempts necessary to establish the $R d l$ allele in generation 1 in three trials.

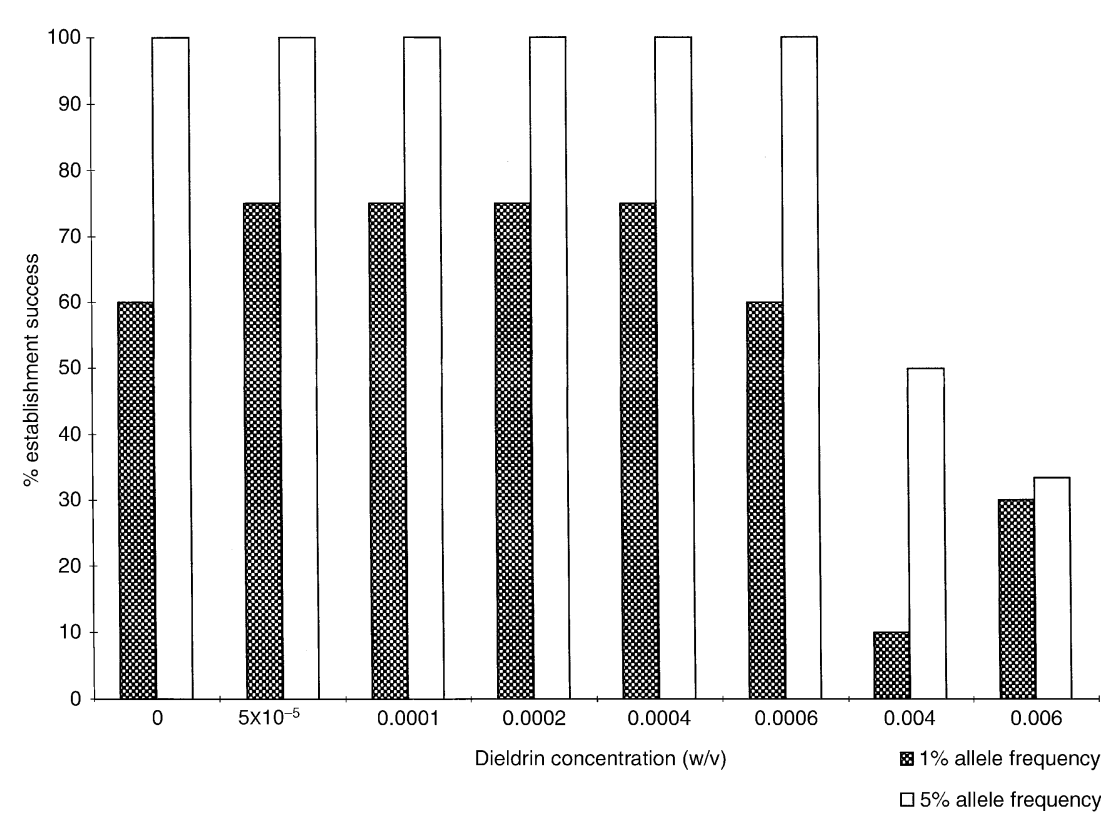


(a)

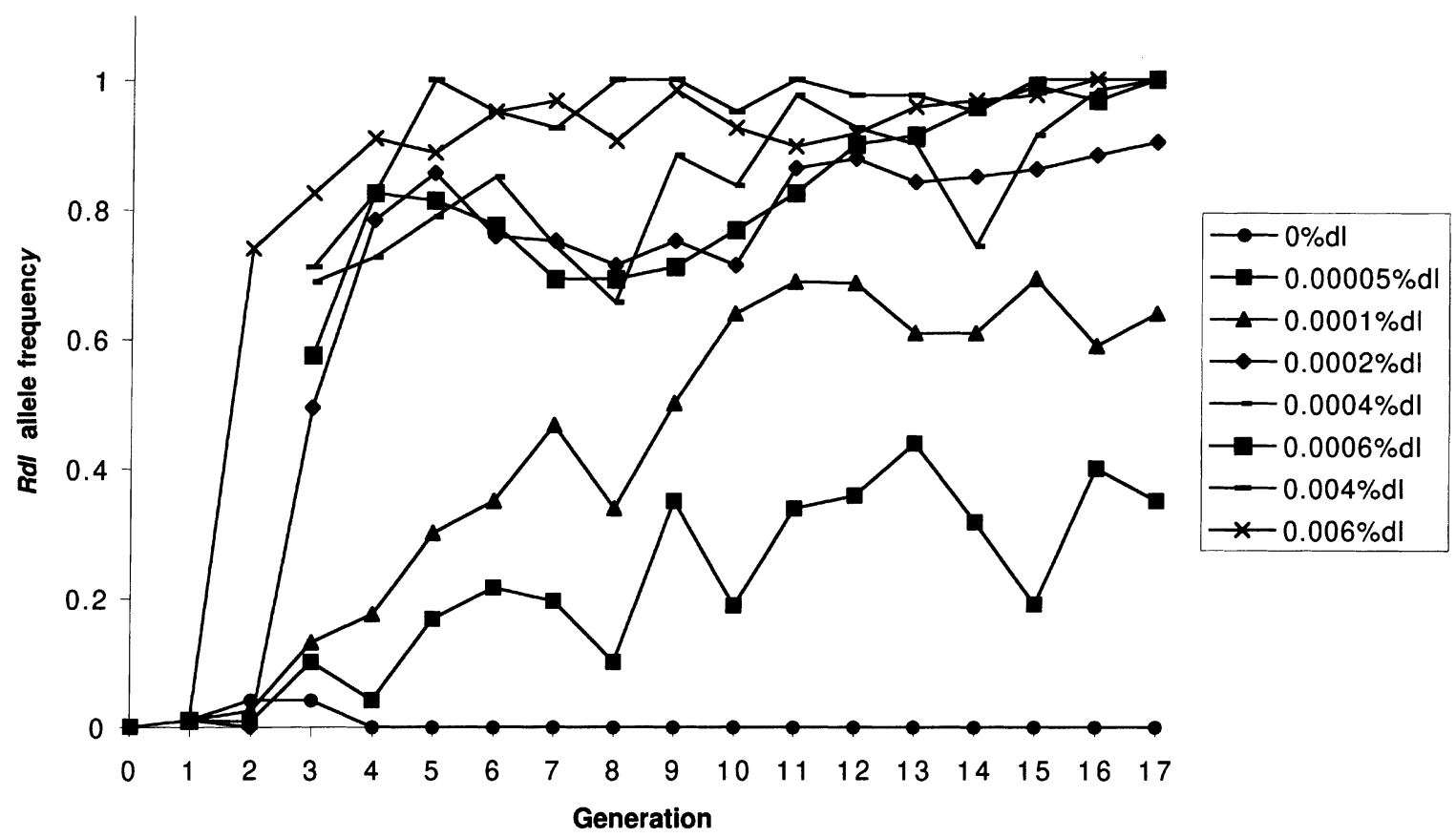

(b)

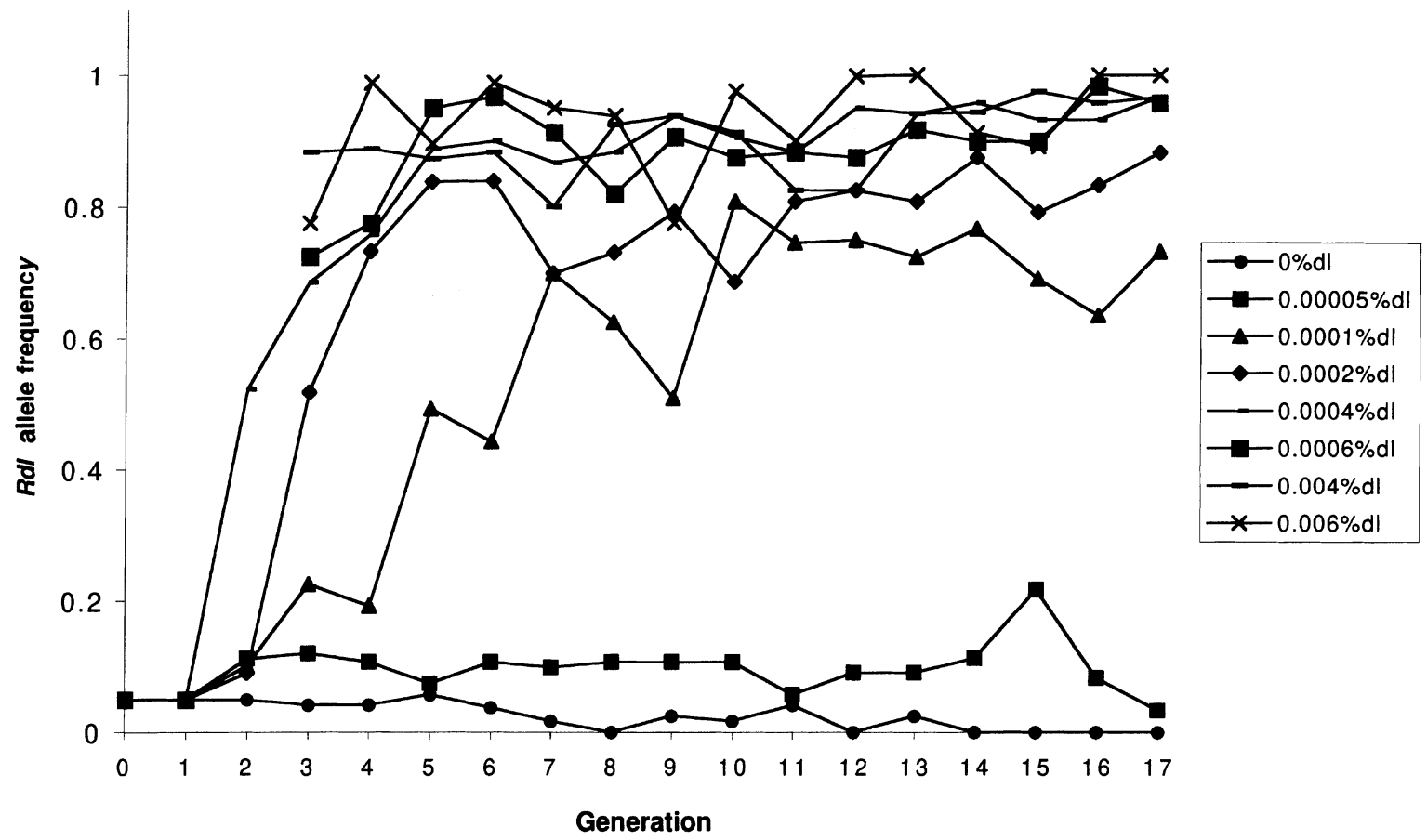

Fig. $2 R d l$ allele frequencies over 17 generations for populations raised on a range of concentrations from 0 to $0.006 \%$ (w/v) dieldrin. Results are averaged over three trials. (a) Initial $R d l$ frequency of $1 \%$. (b) Initial $R d l$ frequency of $5 \%$. 
the summary of results (Fig. 3). Taken as a whole the data are significantly correlated $\left(r=0.92, t_{6}=5.75\right.$, $P<0.002)$.

\section{Discussion}

Whitten \& McKenzie (1982) and Tabashnik (1990) have suggested that random genetic drift may be important in the evolution of resistance even when selection for resistance is intense. This possibility has essentially been ignored in the resistance literature (McKenzie, 1996) and the experiments reported here are the first to test the proposition empirically. The results indicate that random genetic drift is indeed important in the initial establishment of the $R d l$ allele in the populations (Fig. 1). Similar conclusions may be expected for other evolutionary systems. In the current experiments the initiation frequencies of the resistance allele are artificially high compared to those expected in natural populations when resistance first evolves. Depending on what assumptions are made, frequencies of $10^{-3}-10^{-13}$ are initially expected at a resistance locus when a pesticide is first introduced (Whitten \& McKenzie, 1982; McKenzie, 1996). However, even at the elevated frequencies used in the present experiments, the probability of establishment of the $R d l$ allele is consistently lower when the frequency of the allele in the founding population is also lower (Fig. 1). The impact of chance events in natural populations is therefore likely to be even more significant.

The likelihood of establishment is also concentration-dependent (Fig. 1). At higher concentrations of

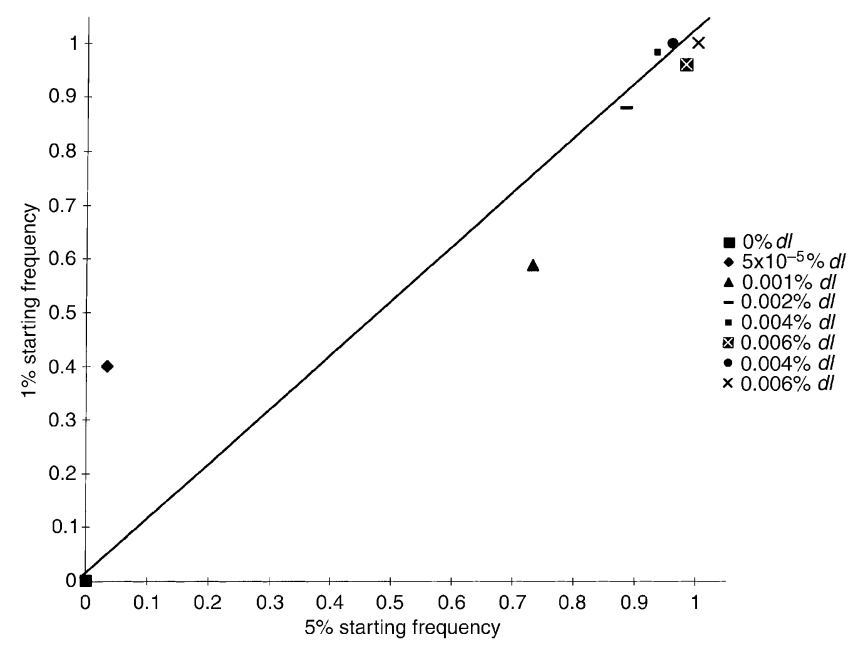

Fig. $3 R d l$ allele frequencies at generation 17 for 1 and $5 \%$ starting allele frequency population cages at different concentrations. Results are averaged over three trials. The line represents the relationship for equivalence of results from the two initiation frequencies. dieldrin the population is unlikely to survive unless the $R d l$ allele is represented in the first generation of insecticide usage because, at these concentrations, susceptibles will not survive. This may have important implications for pest management, depending on population structure and the levels of migration between populations (McKenzie, 1996).

Once the $R d l$ allele is established in a population the evolutionary outcomes are essentially independent of the frequency of the allele in the founding populations (Figs 2 and 3). In the absence of dieldrin the $R d l$ allele is lost from all populations (Fig. 2). This result could be because random genetic drift or selection against resistant phenotypes. The latter explanation is favoured as, in the absence of dieldrin, selection against dieldrinresistant $L$. cuprina has been consistently recorded in previous studies (Whitten et al., 1980; McKenzie, 1990, 1996).

In population cages maintained at concentrations greater than $0.00005 \%(\mathrm{w} / \mathrm{v})$, dieldrin-resistant phenotypes are at a selective advantage and the rate of increase of the $R d l$ allele is a function of concentration (Figs 2 and 3). This observation is also in accord with previous results (McKenzie \& Whitten, 1982).

The results observed in the $0.00005 \%(\mathrm{w} / \mathrm{v})$ dieldrin populations indicate the potential importance of the balance between random genetic drift and selection at low concentrations of an insecticide once the resistant allele is established. In accord with the results at other concentrations, the resistant allele is more likely to establish at the higher founding frequency (Fig. 1). However, at this concentration there is a subsequent increase in frequency of the $R d l$ allele in cages initiated at $1 \% R d l$ (Fig. 2). In cages initiated at $5 \% R d l$ allele frequency, the average allele frequency fluctuates around this value in subsequent generations and one population fixed for the susceptible allele. Clearly the distinction between the relative importance of selection and random genetic drift is extremely subtle at low concentrations of the insecticide.

The present results indicate that if the $R d l$ allele was present in the population it would be selected for at concentrations that are not lethal to susceptibles. Indeed selection for a monogenic response to resistance to dieldrin is possible at trace concentrations of the insecticide, provided the $R d l$ allele is present in the population. Thus, selection for a monogenic response can occur at concentrations that do not control the pest (McKenzie \& Whitten, 1982).

The results support the observation that monogenic responses may occur within selection regimes at concentrations less than the $\mathrm{LC}_{100}$ of susceptibles if an appropriate resistant allele is in the population (Roush \& Hoy, 1981; Heather, 1986; Martin \& McKenzie, 
1990). The likelihood of that occurrence is a function of mutation rate, the population structure, the toxicological phenotypes of resistant and susceptible genotypes, and the relative fitness of those genotypes in the presence, or the absence, of the pesticide. The initial establishment of the resistant allele can also be influenced by random genetic drift (Fig. 1).

In the Australian sheep blowfly, L. cuprina, there is a relative fitness 'cost' associated with being resistant to dieldrin in the absence of the chemical (Whitten et al., 1980; McKenzie, 1990, 1996). Exposure to very low concentrations of the insecticide during development overcomes this. More generally the concentration at which there is a balance between selection for and selection against resistant phenotypes will be specific to a particular system given the variation in fitness costs observed (Bergelson \& Purrington, 1996; McKenzie, 1996).

The same conclusion could be drawn for more general evolutionary phenomena. The genetics of adaptation and the interplay between selection and random genetic drift are best viewed in this context (Orr \& Coyne, 1992).

\section{Acknowledgements}

Dr Phil Batterham is thanked for comments on the manuscript, and Janet Yen for superb technical assistance. The referees are thanked for helpful suggestions. The work was supported by a grant from the Australian Research Council.

\section{References}

BERGElson, J. AND PURRINGTON, C. B. 1996. Surveying patterns in the cost of resistance in plants. Am. Nat., 148, 536-558.

CARRIÈRE, Y., DELAND, J.-P., ROFF, D. A. AND VINCENT, C. 1994. Life-history costs associated with the evolution of insecticide resistance. Proc. R. Soc. B, 258, 35-40.

DALY, J. C., FISK, J. H. AND FORRESTER, N. W. 1988. Selective mortality in field trials between strains of Heliothis armigera (Lepidoptera: Noctuidae) resistant and susceptible to pyrethroids: functional dominance of resistance and age class. J. Econ. Entomol., 81, 1000-1007.

DENHOLM, I., PICKETT, J. A. AND DEVONSHIRE, A. L. (eds), 1999. Insecticide Resistance: from Mechanisms to Management. CABI Publishing, Wallingford.

ENDler, J. A. 1986. Natural Selection in the Wild. Princeton University Press, Princeton, NJ.

FOlletT, P. A., GOUld, F. AND KENNEDY, G. G. 1993. Comparative fitness of three strains of Colorado potato beetle (Coleoptera: Chrysomolidae) in the field: spatial and temporal variation in insecticide selection. J. Econ. Entomol., 86, 1324-1333.

GRESSEL, J. 1995. Catch 22 - Mutually exclusive strategies for delaying/preventing polygenically vs monogenically inherited resistances. In: Ragsdale, N. (ed.) Options 2000, pp. 1-10. ACS, Washington, DC.

HEATHER, N. W. 1986. Sex-linked resistance to pyrethroids in Sitophilus oryzae (L.) (Coleoptera: Curculionidae). J. Stored Prod. Res., 22, 15-20.

KIMURA, M. 1983. The Neutral Theory of Molecular Evolution. Cambridge University Press, Cambridge.

MACNAIR, M. R. 1991. Why the evolution of resistance to anthropogenic toxins normally involves major gene changes: the limits to natural selection. Genetica, 84, 213-219.

MARTIN, P. J. AND McKENZIE, J. A. 1990. Levamisole resistance in Trichostrongylus colubriformis: a sex-linked recessive character. Int. J. Parasitol., 20, 867-872.

McKENZIE, J. A. 1990. Selection at the dieldrin resistance locus in overwintering populations of Lucilia cuprina (Wiedemann). Aust. J. Zool., 38, 493-501.

McKenZIE, J. A. 1996. Ecological and Evolutionary Aspects of Insecticide Resistance. R. G. Landes and Academic Press, Austin, TX.

McKenZiE, J. A. AND BATterham, P. 1994. The genetic, molecular and phenotypic consequences of selection for insecticide resistance. Trends Ecol. Evol., 9, 166-169.

McKenZIE, J. A. AND BATTERhAM, P. 1998. Predicting insecticide resistance: mutagenesis, selection and response. Phil. Trans. R. Soc. B, 335, 1729-1734.

McKenZIE, J. A. AND WhitTEN, M. J. 1982. Selection for insecticide resistance in the Australian sheep blowfly, Lucilia cuprina. Experientia, 38, 84-85.

ORR, H. A. AND COYNE, J. A. 1992. The genetics of adaptation: a reassessment. Am. Nat., 140, 725-742.

ROUSH, R. T. AND HOY, M. A. 1981. Laboratory, glasshouse and field studies of artificially selected carbaryl resistance in Metaseiulus Occidentalis. J. Econ. Entomol., 74, 142-147.

ROUSH, R. T. AND McKENZIE, J. A. 1987. Ecological genetics of insecticide and acaricide resistance. Ann. Rev. Ent., 32, 361-380.

SMYTH, K.-A., PARKER, A. G., YEN, J. L. AND McKENZIE, J. A. 1992. Selection of dieldrin-resistant strains of Lucilia cuprina R.-D. (Diptera: Calliphoridae) following ethyl methanesulfonate mutagenesis of a susceptible strain. J. Econ. Entomol., 85, 352-358.

TABASHNIK, B. E. 1990. Modelling and evolution of resistance management tactics. In: Roush, R. T. and Tabashnik, B. C. (eds) Pesticide Resistance in Arthropods, pp. 153-182. Chapman \& Hall, New York.

WhitTEN, M. J. AND McKenZIE, J. A. 1982. The genetic basis for pesticide resistance. In: Lee, K. E. (ed.) Proceedings of the 3rd Australasian Conference on Grassland Invertebrates, pp. 1-16. S.A. Govt. Printer, Adelaide.

WhitTen, M. J., DEARn, J. M. AND McKenZIE, J. A. 1980. Field studies on insecticide resistance in the Australian sheep blowfly, Lucilia cuprina. Aust. J. Biol. Sci., 33, 725-735. 